\title{
Discreteness-Induced Oscillatory Instabilities of Dark Solitons
}

\author{
Magnus Johansson ${ }^{1}$ and Yuri S. Kivshar ${ }^{2}$ \\ ${ }^{1}$ Department of Physics and Measurement Technology, Linköping University, S-581 83 Linköping, Sweden \\ ${ }^{2}$ Optical Sciences Center, Research School of Physical Sciences and Engineering, The Australian National University, \\ Canberra ACT 0200, Australia
}

(Received 18 August 1998)

\begin{abstract}
We reveal that even weak inherent discreteness of a nonlinear model can lead to instabilities of the localized modes it supports. We present the first example of an oscillatory instability of dark solitons, and analyze how it may occur for dark solitons of the discrete nonlinear Schrödinger and generalized Ablowitz-Ladik equations. [S0031-9007(98)08088-0]
\end{abstract}

PACS numbers: 42.65.Tg, 05.45.Yv, 63.20.Pw

Wave instabilities are probably the most remarkable nonlinear phenomena that may occur in nature [1]. One of the first instabilities discovered for nonlinear models was the modulational instability, which is known to be an effective physical mechanism in fluids [2] and optics [3] for breakup of continuous modes into solitary waves. Also, the solitary waves themselves may become unstable, and the analysis of their instabilities is an important problem of nonlinear physics. Instabilities are known to occur for both bright [4] and dark [5] solitary waves of different nonintegrable nonlinear models.

Recently, a new type of solitary-wave instability, oscillatory instability, has been found to occur for bright Bragg gap solitons in the generalized Thirring model [6]. Such an instability is characterized by complex eigenvalues, and its scenario is associated with a resonance between the long-wavelength radiation and soliton internal modes which appear in the soliton spectrum when the model becomes nonintegrable [7]. In spite of the fact that oscillatory instabilities appear often in dissipative models [8], their manifestation in continuous Hamiltonian models is rare [9], and so far no example has been known for oscillatory instability of dark solitons.

The aim of this Letter is twofold. First, we analyze what we believe to be the first examples of oscillatory instabilities of dark solitons, by considering the important cases of the discrete nonlinear Schrödinger (DNLS) and generalized Ablowitz-Ladik (AL-DNLS) models. We reveal two different scenarios for the dark-soliton oscillatory instability, which may occur due to either a resonance between radiation modes and the soliton internal mode, or a resonance between two soliton internal modes. Second, we demonstrate that even a weak inherent discreteness may drastically modify the dynamics of a nonlinear system leading to instabilities which have no analog in the continuum limit.

First, we consider the well-known DNLS equation,

$$
i \dot{\psi}_{n}+C\left(\psi_{n+1}+\psi_{n-1}\right)+\left|\psi_{n}\right|^{2} \psi_{n}=0,
$$

where the dot stands for the derivative in time. Stationary localized solutions of Eq. (1) in the form $\psi_{n}(t)=\phi_{n} e^{i \Lambda t}$, where $\Lambda=2 C \cos k+\left(\phi^{(0)}\right)^{2}$, may exist as dark-soliton modes with the nonvanishing boundary conditions $\phi_{n} \rightarrow$ $\pm \phi^{(0)} e^{i k n}(n \rightarrow \pm \infty)$, provided the background wave is modulationally stable, i.e., for $C \cos k<0$ [10]. Without loss of generality, we can put the background intensity to unity, $\phi^{(0)}=1$.

The structure of the dark-soliton modes of Eq. (1) has been discussed earlier [11,12]. Here, we consider the case $C>0$, in which the background wave has $k=\pi$ and is "staggered," as shown in Figs. 1(a) and 1(b). The transformation $\psi_{n} \rightarrow(-1)^{n} \psi_{n}$ immediately yields the corresponding "unstaggered" modes $(k=0)$ for negative $C$.

The dark-soliton modes presented in Figs. 1(a) and 1(b) describe two types of stationary "black" solitons [5] in a discrete lattice, the on-site mode ( $A$ mode) centered with zero intensity at a lattice site, and the intersite mode ( $B$ mode) centered between two sites. These two modes can be uniquely followed from the continuous limit $(C \rightarrow \infty)$ to the "anticontinuous" limit $(C=0)$. At $C=0$, the $A$ mode takes the form $\phi_{n}=$ $(\ldots,-1,+1,-1,0,+1,-1,+1, \ldots)$, and it describes a single "hole" in a background wave with constant amplitude and a $\pi$ phase shift across the hole. Similarly, the $B$ mode takes the form $\phi_{n}=(\ldots,-1,+1,-1,-1,+1$, $-1, \ldots)$, and it describes the lattice oscillation mode with a $\pi$ phase shift between two neighboring sites and no hole.

Linear stability of the $A$ mode for small enough $C$ follows from Aubry's theorem (Theorem 9 in Ref. [13])

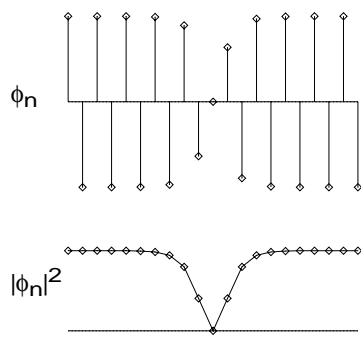

(a)
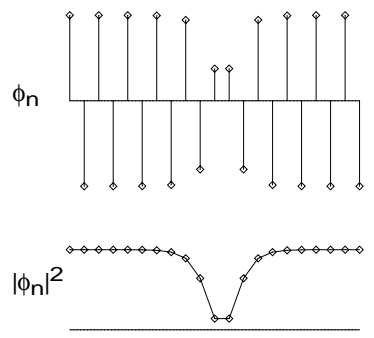

(b)
FIG. 1. (a), (b) Two types of "staggered" dark-soliton modes in a lattice. Shown are the oscillation amplitude at each site (upper row) and the value $\left|\psi_{n}\right|^{2}$ (lower row). 
relating the linear stability of multibreathers to the extrema of the effective action which is a function of the relative phases $\alpha_{n}$ of the single breathers. For the DNLS equation (1) with a positive nonlinearity, local minima of the effective action correspond to stable solutions. A perturbative expression of the effective action to order $C^{2}$ was obtained in Eq. (A10) in [14]. For small $C$, it is enough to consider the phase interactions between nearest and next-nearest neighboring sites. Then, the lowest order contribution to the effective action from neighboring excited sites is $\sum_{n}\left[2 C|\Lambda| \cos \left(\alpha_{n+1}-\alpha_{n}\right)-C^{2} \cos ^{2}\left(\alpha_{n+1}-\right.\right.$ $\left.\alpha_{n}\right)$ ], while the contribution from the next-nearestneighbor interaction is $-C^{2} \sum_{n \neq n_{0}} \cos \left(\alpha_{n+1}-\alpha_{n-1}\right)+$ $2 C^{2} \cos \left(\alpha_{n_{0}+1}-\alpha_{n_{0}-1}\right)$, where $n_{0}$ is the site with the "hole." For the $A$ mode, $\alpha_{n+1}-\alpha_{n}=\pi$, $\alpha_{n+1}-\alpha_{n-1}=0, \quad n \neq n_{0}, \quad \alpha_{n_{0}+1}-\alpha_{n_{0}-1}=\pi$, so that the effective action has a local minimum and the mode is stable for small $C$.

For the $B$ mode, there are two neighboring sites having the same phase at the center, which suggests that the corresponding extremum of the effective action is a saddle. Numerical investigation of the eigenvalue problem also shows that the $B$ mode is unstable for all $C$.

Let us consider the linear stability of the dark-soliton modes for nonvanishing $C$. Assuming $\psi_{n}(t)=\left[\phi_{n}+\right.$ $\left.\epsilon_{n}(t)\right] e^{i \Lambda t}$, we obtain the linearized equations for $\epsilon_{n}$

$$
\begin{aligned}
i \dot{\epsilon}_{n}+C\left(\epsilon_{n+1}+\epsilon_{n-1}\right)+2\left|\phi_{n}\right|^{2} \epsilon_{n} & \\
+\phi_{n}^{2} \epsilon_{n}^{*}-\Lambda \epsilon_{n} & =0 .
\end{aligned}
$$

Writing $\epsilon_{n}=\xi_{n}+i \eta_{n}$ for real $\phi_{n}$ yields

$$
\frac{d}{d t}\left(\begin{array}{c}
\xi_{n} \\
\eta_{n}
\end{array}\right)=\left(\begin{array}{cc}
0 & H^{+} \\
-H^{-} & 0
\end{array}\right)\left(\begin{array}{l}
\xi_{n} \\
\eta_{n}
\end{array}\right) \equiv \hat{M}\left(\begin{array}{l}
\xi_{n} \\
\eta_{n}
\end{array}\right),
$$

where for a system of $N$ sites $\hat{M}$ is a $2 N \times 2 N$ matrix and $\mathrm{H}^{+}$and $\mathrm{H}^{-}$are $\mathrm{N} \times \mathrm{N}$ matrices with time-independent coefficients, $H_{i j}^{ \pm}=\left[\Lambda-(2 \mp 1) \phi_{i}^{2}\right] \delta_{i, j}-C\left(\delta_{i, j+1}+\right.$ $\delta_{i, j-1}$ ) (boundary conditions not explicitly taken into account). Linear stability is then equivalent to the matrix $\hat{M}$ having all its eigenvalues on the imaginary axis.

When $C=0$, all eigenvalues of $\hat{M}$ lie at zero except, for the $A$ mode, one complex conjugated pair at $\pm i$ corresponding to a mode localized on the hole. When $C$ is increased, the eigenvalues at zero spread on the imaginary axis creating a phonon band of extended states, which corresponds to a continuous spectrum for large $N$. Assuming $\epsilon_{n}=a e^{i(\kappa n-\omega t)}+b e^{-i(\kappa n-\omega t)}$ yields the dispersion relation, $\omega= \pm \sqrt{16 C^{2} \cos ^{4}(\kappa / 2)+8 C \cos ^{2}(\kappa / 2)}$, so that the eigenvalues corresponding to the continuous band lie between 0 (at $\kappa=\pi$ ) and $\pm i \sqrt{16 C^{2}+8 C}$ (at $\kappa=0$ ). On the other hand, the pair of eigenvalues at $\pm i$ will move towards zero on the imaginary axis as $C$ is increased. For small $C$, we can assume that the corresponding eigenmode is almost completely localized at the hole, so that $\epsilon_{n_{0}+1} \approx \epsilon_{n_{0}-1} \approx 0$. Since $\phi_{n_{0}}=0$, we obtain that this mode oscillates with frequency $\Lambda=1-2 C$, so that the corresponding eigenvalues are $\pm i(1-2 C)$, to the low- est order in $C$. Then, equating this result with the expression above for the edge of the continuous band gives an estimate to the value of $C$ where these two eigenvalues coincide: $C=1 / \sqrt{3}-1 / 2 \approx 0.07735$. This agrees quite well with the exact, numerically obtained value $C \equiv C_{\mathrm{cr}} \approx 0.07647$. At $C=C_{\mathrm{cr}}$, a Hopf-type bifurcation occurs, as two complex conjugated pairs of eigenvalues leave the imaginary axis and go out in the complex plane (see Fig. 2). Thus, an oscillatory instability occurs for the $A$ mode when $C>C_{\mathrm{cr}}$, with the instability growth rate $\lambda$ given by the real part of the unstable eigenvalue (see Fig. 2). For the $B$ mode, all eigenvalues lie at zero when $C=0$. As soon as $C$ is increased, one pair goes out on the real axis and stays there for all $C>0$. Thus, the $B$ mode is always unstable.

For $C>C_{\mathrm{cr}}$, the imaginary part of the unstable eigenvalue moves towards zero (i.e., the oscillation frequency decreases) as $C$ is increased. The real part of the eigenvalue vs $C$ is shown in Fig. 2 for two different system sizes, and compared with estimations of the maximum growth rate from direct numerical integration of the DNLS model for chains large enough to eliminate the influence of the boundaries. In all cases the instability is largest for $C \approx 0.32$ where $\lambda \approx 0.120$. For larger $C$, there appear stable "windows" for the finite systems, where the mode is stabilized by the boundaries. The location of these windows depends critically on the size of
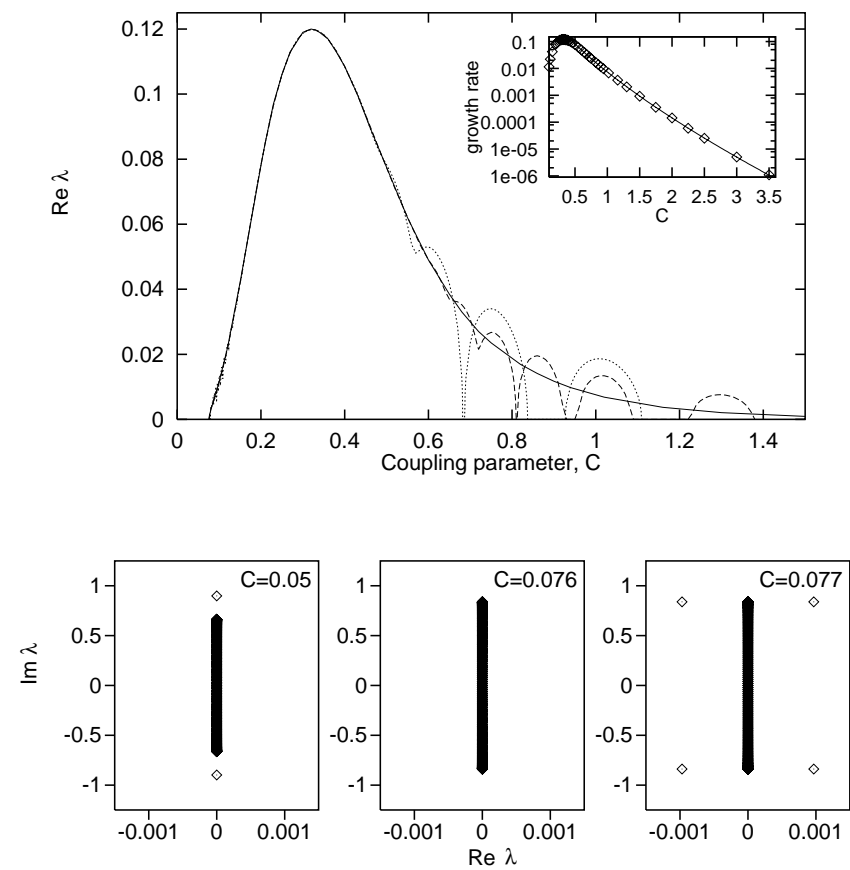

FIG. 2. Real part of the unstable eigenvalues as a function of $C$ in the model (1) for $N=61$ (dotted) and $N=121$ (dashed) sites, and the growth rate (solid) for an infinite system, calculated for lattices of up to 15000 sites. Inset: Asymptotic fit of the growth rate (points) with stretched exponential (line) (see text). Below: Instability scenario shown as the evolution of the eigenvalues of $\hat{M}$ for $N=121$ sites. 
the system and the boundary conditions (in Fig. 2, periodic boundary conditions $\psi_{N+1}=\psi_{1}$ are used).

Similar "reentrant instabilities" have been found for discrete breathers of the finite-width Klein-Gordon chains [15]. Qualitatively, the explanation is that for small systems, the eigenvalues corresponding to the "continuous" spectrum are rather sparsely distributed, and the corresponding eigenvectors are localized over the size of the system. Thus, as the eigenvalue corresponding to the unstable mode approaches the imaginary axis when increasing $C$, it may find a hole in the spectrum and join the imaginary axis for a while. Then, a further increase of $C$ causes a collision with the next imaginary eigenvalue, and a new instability occurs. This procedure repeats itself until the eigenvalue has passed the phonon band eigenvalue which is closest to zero. After this the state is stable for all larger values of $C$ (e.g., in Fig. 2 the state is stable for all $C>1.11$ when $N=61$, and for all $C>1.38$ when $N=121$ ). Increasing the system size implies that the eigenvalue distribution on the imaginary axis will be more dense, so that the stable windows will be smaller and completely disappear in the limit of the infinite system, where the unstable eigenvalue never joins the imaginary axis.

For an infinite DNLS chain, the instability growth rate decreases in an exponential-like way to zero for large $C$, and thus indicates that the dark mode is unstable for all $C>C_{\mathrm{cr}}$. A quite good asymptotic fit is obtained with a stretched exponential, $\lambda \sim \exp \left(-b C^{\gamma}\right)$, with $\gamma \approx 0.7$ (see inset in Fig. 2). We also investigated the case of varying $\phi^{(0)}$ to keep the complementary norm [5] constant when varying $C$. In that case, we found the asymptotic decay of the instability growth rate with $C$ to be faster than purely exponential.

Figure 3 shows the time evolution for two different values of $C$ when the initial state is a slightly perturbed dark $A$ mode. A small perturbation has been
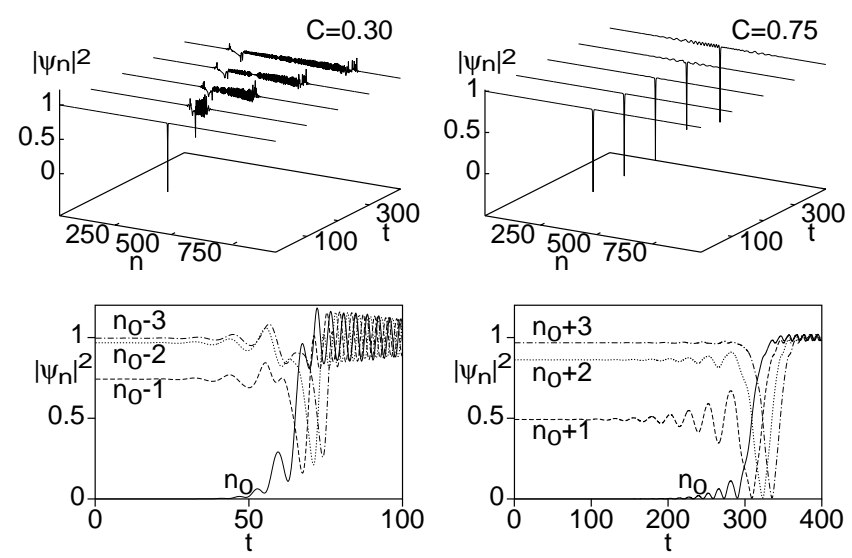

FIG. 3. Time evolution of $\left|\psi_{n}\right|^{2}$ with slightly perturbed dark $A$ modes as initial conditions for $C=0.30$ (left) and $C=0.75$ (right). Lower figures show the detailed dynamics for a few sites around the center. chosen to be approximately in the direction of the unstable eigenvector. If instead a random perturbation is chosen, the qualitative behavior is the same, except that there will be an initial small amount of radiation before the unstable internal eigenmode gets excited. The unstable eigenvector is always spatially symmetric around the central site, and therefore antisymmetric with respect to the dark mode itself, since the latter is antisymmetric. Thus, the main effect of the instability is a transition from a black (zero intensity at the middle) to grey (nonzero intensity at the middle) soliton, as can be clearly identified at least for $C>0.3$. This is similar to the instability scenario of dark solitons in some continuous models [5], except the oscillatory dynamics. The direction of the resulting grey soliton is determined by the sign of the perturbation projected on the unstable eigenmode. For large $C$, the grey soliton is almost black, moves slowly, and the radiation is small, while for smaller $C$ the minimum value of the soliton intensity increases as well as its velocity and the amount of radiation. For small and intermediate values of the coupling parameter $C$, the resulting grey soliton decays continuously into radiation (see Fig. 3).

It is important to study the oscillatory instability of dark solitons for other types of nonlinear lattices. Here, we consider the AL-DNLS equation $i \dot{\psi}_{n}+C\left(\psi_{n+1}+\psi_{n-1}\right)+$ $\mu\left|\psi_{n}\right|^{2} \psi_{n}+\frac{1}{2}(\mu-1)\left|\psi_{n}\right|^{2}\left(\psi_{n+1}+\psi_{n-1}\right)=0$, where $0 \leq \mu \leq 1$. The case $\mu=0$ corresponds to the integrable AL model, whereas the case $\mu=1$, to the DNLS model analyzed above.

With the general form $\psi_{n}(t)=\phi_{n} e^{i \Lambda t}$, and boundary conditions $\phi_{n} \rightarrow \pm \phi^{(0)} e^{i k n}(n \rightarrow \pm \infty)$, the frequency $\Lambda$ is determined by $\Lambda=2 C \cos k+\left(\phi^{(0)}\right)^{2}[\mu+(\mu-$ 1) $\cos k]$. In particular, for the black mode $(k=\pi)$ the relation becomes $\Lambda=-2 C+\left(\phi^{(0)}\right)^{2}$, just as for the DNLS model. As above, we put $\phi^{(0)}=1$ without loss of generality.

In the AL-DNLS model, there is a lower limit of $C$ for the existence of dark solitons, due to the instability of the background when the effective coupling changes sign (see, e.g., Ref. [16]). This occurs when $C+(\mu-$ 1) $\left(\phi^{(0)}\right)^{2} / 2=0$, so that, when $\phi^{(0)}=1$, the dark modes exist only for $C>(1-\mu) / 2$ (Fig. 4, dashed line).

Considering $\psi_{n}(t)=\left[\phi_{n}+\epsilon_{n}(t)\right] e^{i \Lambda t}$ as above, we obtain the linearized equations for the small perturbation $\epsilon_{n}$, and the dispersion relation for the continuous spectrum (for the staggered mode with $\lim _{|n| \rightarrow \infty} \phi_{n}^{2}=$ 1): $\omega= \pm 2 \sqrt{\nu(\kappa)[1+\nu(\kappa)]}$, where $\nu(\kappa) \equiv(2 C+$ $\mu-1) \cos ^{2}(\kappa / 2)$. Therefore, the eigenvalues corresponding to the continuous band lie between 0 (at $\kappa=$ $\pi)$ and $\pm i 2 \sqrt{(2 C+\mu-1)(2 C+\mu)}($ at $\kappa=0)$ for $C>(1-\mu) / 2$.

As above, an approximate expression for the onset of instability can be obtained by assuming that the internal mode is completely localized at the hole, so that $\epsilon_{n_{0}+1} \approx \epsilon_{n_{0}-1} \approx 0$. Then, the corresponding eigenvalues 


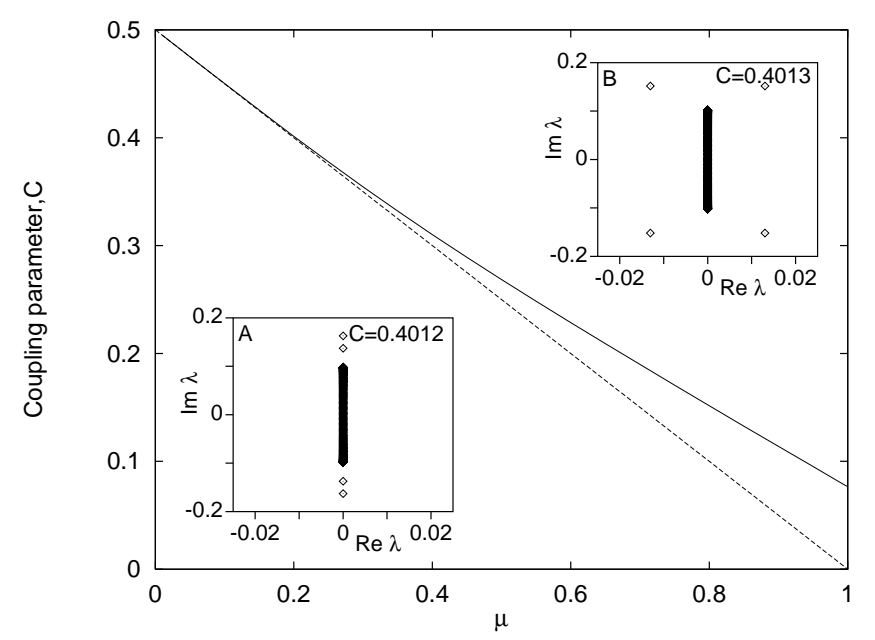

FIG. 4. Solid line: Numerically obtained instability threshold $C_{\mathrm{cr}}(\mu)$ for the AL-DNLS model. Dashed line: Minimum value of $C$ for the existence of the dark localized modes. Insets: Examples of the eigenvalues at $\mu=0.2$, below (A) and above (B) the threshold curve $C_{\mathrm{cr}}(\mu)$.

are $\pm i(1-2 C)$, to the lowest order in $C$. The collision with the phonon band edge is then expected to occur for $C \approx(1-4 \mu) / 6+\sqrt{\mu^{2}+\mu+1} / 3$. Comparing this result with the numerically obtained values of $C$ for the bifurcation (see Fig. 4) shows that the instability for smaller $\mu$ occurs for smaller $C$ than expected from this analytical estimate. The reason why the bifurcation occurs earlier is that it in fact results not from a collision with the band edge phonon, but with a second localized mode that has bifurcated from the band edge slightly before (see the insets in Fig. 4). As a matter of fact, studying the bifurcation for the DNLS model very closely shows that also in this case the collision probably occurs with a second localized mode coming from the phonon band. However, this localized mode only occurs extremely close to the bifurcation when $\mu$ is close to 1 (for $\mu=1$ we observe it for $C>0.076464$, and the bifurcation occurs at $C=0.076468 \ldots$.). And since it will be very weakly localized, we are not able to say for sure whether it will be localized or not for the infinite system. But for smaller $\mu$, this localized mode undoubtedly exists, and has the same spatial symmetry as the hole mode. It describes an internal degree of freedom of the dark soliton, i.e., its internal mode.

Otherwise, the qualitative scenario with stable and unstable windows for finite systems and instability all the way up to the continuum limit for the infinite system remains the same for all AL-DNLS lattices with $0<\mu \leq$ 1. The exception is the limit of the AL model $(\mu=0)$ which is known to be exactly integrable. At $\mu=0$, dark solitons are always stable for all $C$ and their spectrum has a pair of eigenvalues exactly at zero corresponding to the exact translational invariance.

In conclusion, we have described, for the first time to our knowledge, the oscillatory instability of dark solitons. This new type of dark-soliton instability appears due to inherent discreteness of a nonlinear lattice model, and the universality of the instability scenario suggests that it should be also observed in other nonlinear models supporting dark solitons. We remark that the instability observed here may be regarded as an extension of the instabilities existing for small lattices [17].

M. J. acknowledges support from the Swedish Natural Science Research Council. Yu. K. acknowledges a partial support from the Australian Research Council.

[1] E. Infeld and G. Rowlands, Nonlinear Waves, Solitons, and Chaos (Cambridge University Press, Cambridge, 1990).

[2] T.B. Benjamin and J.E. Feir, J. Fluid Mech. 27, 417 (1967).

[3] K. Tai, A. Hasegawa, and A. Tomita, Phys. Rev. Lett. 56, 135 (1986).

[4] See, e.g., E. A. Kuznetsov, A.M. Rubenchik, and V.E. Zakharov, Phys. Rep. 142, 103 (1986).

[5] See, e.g., Yu. S. Kivshar and B. Luther-Davies, Phys. Rep. 298, 81 (1998).

[6] I. V. Barashenkov, D.E. Pelinovsky, and E. V. Zemlyanaya, Phys. Rev. Lett. 80, 5117 (1998); A. De Rossi, C. Conti, and S. Trillo, Phys. Rev. Lett. 81, 85 (1998).

[7] Yu. S. Kivshar et al., Phys. Rev. Lett. 80, 5032 (1998).

[8] See, e.g., D. Walgraef, Spatio-Temporal Pattern Formation (Springer-Verlag, New York, 1997).

[9] See, e.g., R. L. Pego, P. Smereka, and M.I. Weinstein, Nonlinearity 8, 921 (1995).

[10] Yu.S. Kivshar and M. Peyrard, Phys. Rev. A 46, 3198 (1992).

[11] Yu.S. Kivshar, W. Królikowski, and O. A. Chubykalo, Phys. Rev. E 50, 5020 (1994).

[12] D. Hennig et al., Phys. Rev. E 54, 5788 (1996).

[13] S. Aubry, Physica (Amsterdam) 103D, 201 (1997).

[14] M. Johansson and S. Aubry, Nonlinearity 10, 1151 (1997).

[15] J. L. Marín and S. Aubry, Physica (Amsterdam) 119D, 163 (1998).

[16] V. V. Konotop and M. Salerno, Phys. Rev. E 55, 4706 (1997); 56, 3611 (1997).

[17] J. Carr and J.C. Eilbeck, Phys. Lett. 109A, 201 (1985); J. C. Eilbeck, P.S. Lomdahl, and A.C. Scott, Physica (Amsterdam) 16D, 318 (1985); J. C. Eilbeck and A.C. Scott, in Structure, Coherence and Chaos in Dynamical Systems, edited by P.L. Christiansen and R. D. Parmentier (Manchester University Press, Manchester, 1989), p. 139. 